Homology, Homotopy and Applications, vol.10(2), 2008, pp.59-81

\title{
PARTIAL MAGMATIC BIALGEBRAS
}

\author{
EMILY BURGUNDER AND RALF HOLTKAMP
}

(communicated by Luchezar Avramov)

\begin{abstract}
A partial magmatic bialgebra, or $(T ; S)$-magmatic bialgebra, where $T \subset S$ are subsets of $\mathbb{N}_{\geqslant 2}$, is a vector space endowed with an $n$-ary operation for each $n \in S$ and an $m$-ary co-operation for each $m \in T$ satisfying some compatibility and unitary relations. We prove an analogue of the Poincaré-Birkhoff-Witt theorem for these partial magmatic bialgebras.
\end{abstract}

\section{Introduction}

Let $\mathcal{H}$ be a cocommutative bialgebra over a field $\mathbb{K}$ of characteristic zero. Then the Poincaré-Birkhoff-Witt and the Cartier-Milnor-Moore theorem $[\mathbf{4}, \mathbf{1 2}]$ can be rephrased as:

Theorem 1.1 ([PBW, CMM]). The following are equivalent:

(i) $\mathcal{H}$ is connected.

(ii) $\mathcal{H}$ is isomorphic to $U($ Prim $\mathcal{H})$.

(iii) $\mathcal{H}$ is cofree among the connected cofree coalgebras.

The space of primitive elements Prim $\mathcal{H}$ of $\mathcal{H}$ functorially admits a $\mathcal{L}$ ie-algebra structure, and $U$ is the universal enveloping algebra functor.

This structure theorem has been extended to connected associative bialgebras by J.-L. Loday and M. Ronco in [10], and has led to many other structure theorems for generalised bialgebras (see, for example, $[\mathbf{7}, \mathbf{8}, \mathbf{1 2}, \mathbf{1 3}, \mathbf{1 4}, \mathbf{1 5}]$ ).

There exists now a general theory for these structure theorems for non-unital bialgebras over operads due to J.-L. Loday; see [9]. These bialgebra types are given by operads $\mathcal{A}$ handling the algebra structure, operads $\mathcal{C}$ handling the coalgebra structure, and the compatibility relations between the two structures. This theory gives conditions for the existence of a primitive operad such that an analogue of the structure theorem holds. Even when the conditions are verified, one of the main difficulties remains in unravelling the algebraic structure of the primitives.

Motivated by a systematic study of the theory, the first paradigmatic example of a bialgebra type to consider is the case where both operads $\mathcal{A}$ and $\mathcal{C}$ are free. We call

Received September 11, 2007, revised April 10, 2008; published on July 22, 2008.

2000 Mathematics Subject Classification: 18D50, 16W30.

Key words and phrases: generalized bialgebra, Hopf algebra, Cartier-Milnor-Moore theorem, Poincaré-Birkhoff-Witt theorem, operad, magma, non-associative algebra.

This article is available at http://intlpress.com/HHA/v10/n2/a3

Copyright (c) 2008, International Press. Permission to copy for private use granted. 
this bialgebra type the partial magmatic bialgebras, and study them in this article, continuing some works of both authors $[\mathbf{2}, \mathbf{3}, \mathbf{7}, \mathbf{8}]$, which consider certain bialgebra types with at least one free operad $M a g$ - or $M a g \underline{\underline{n}}$ - with free generators up to arity $n$. Magmatic bialgebras defined over $\mathcal{A}=\mathcal{C}=M a g^{\underline{n}}$ were introduced and classified, over the operad $\mathcal{V}$ ect of primitives, by the first author in [3]. The natural generalisation of the case of magmatic bialgebras over $\mathcal{C}=\operatorname{Mag}^{\underline{m}}, \mathcal{A}=\operatorname{Mag}^{\underline{n}}$, for $m \neq n$, was posed as an open problem in $[\mathbf{3}]$.

More generally, we consider in the first part of the paper $(T ; S)$-magmatic bialgebras, where $T \subset S \subset \mathbb{N}$. Such a $(T ; S)$-magmatic bialgebra is a vector space endowed with several not necessarily (co)associative operations and co-operations, namely one $n$-ary operation for each $n \in S$ and one $m$-ary co-operation for each $m \in T$. The given bialgebra type verifies the conditions of $[\mathbf{9}]$ if the operations and co-operations are supposed to verify a compatibility relation, namely the partial magmatic compatibility relation. The primitive operads $\mathrm{Mag}_{\text {root } \backslash T}^{S}$ that we unravel are free operads generated by infinitely many generators. An equivalent way of defining the operad $M a g_{\text {root } \backslash T}^{S}$ is as $\mathbb{K} \operatorname{Id} \oplus \mathcal{I}$, where $\mathcal{I}$ is a right operadic ideal of the operad $M^{2} g^{S}$. The structure theorem holds for $(T ; S)$-magmatic bialgebras, with $\mathcal{L}$ ie replaced by $M a g_{\text {root } \backslash T}^{S}$.

The second part of the article is to adapt this theorem to the unital framework. In order to stay in the operadic framework, we have to restrict our study to partial magmatic bialgebras, where $S=\{2, \ldots, n\}$ and $T=\{2, \ldots, m\}$.

The question is how the statements of the first part have to be modified so that they are still valid in the presence of a unit. We give an answer to this question by finding the precise compatibility relation that one has to take, and, by the way, answer the open question of $[\mathbf{3}]$.

Since the composition of operations may no longer be associative, the unital framework for general partial magmatic bialgebras needs a more general setting than the operadic setting.

In Section 2 we recall the basics on planar trees, and we introduce $S$-magmatic algebras and $T$-magmatic coalgebras. From Section 3 on, we work with (non-symmetric) operads. We show that $S$-magmatic algebras and $S$-magmatic coalgebras are defined as (co)algebras over operads $\mathrm{Mag}^{S}$. A formula to compute the dimension series of $M a g^{S}$ is given in Proposition 3.8.

In Section 4, we introduce $(T ; S)$-magmatic bialgebras where $T \subset S \subset \mathbb{N}$, and we pay special attention to the case $S=T$. The operads $M a g_{\text {root } \backslash T}^{S}$ are constructed in Section 5 . They are given (in arity $\geqslant 2$ ) by a right operadic ideal (defined analogously to a right ideal in a ring). We also construct related forgetful and universal enveloping functors.

In Section 6, we extend results of $[\mathbf{3}]$ to the case of $(S ; S)$-magmatic bialgebras, and then follow ideas of $[\mathbf{1 0}]$ to prove the main theorem, classifying $(T ; S)$-magmatic bialgebras. In Section 7 we give all necessary modifications of the previous constructions and the structure theorem so that they are still valid in the presence of a unit, provided $S=\{2, \ldots, n\}$ and $T=\{2, \ldots, m\}$.

The structure theorems lead to some pretty combinatorics for the generating series of the corresponding operads. Some typical examples are given in Section 8 . 


\section{2. (T;S)-magmatic bialgebras}

In this section we define $S$-magmatic algebras and $T$-magmatic coalgebras in order to construct $(T ; S)$-magmatic bialgebras.

\section{1. $S$-magmatic algebras}

Definition 2.1. Let $S$ be a subset of $\mathbb{N}_{\geqslant 2}=\{2,3, \ldots\}$. An $S$-magmatic algebra $A$ is a vector space endowed with an $n$-ary operation $\mu_{n}$ for each $n \in S$.

Definition 2.2. A planar tree $T$ is a planar graph, which is assumed to be simple (no loops nor multiple edges), connected, rooted and reduced (no vertices with only one outgoing edge). The set of planar trees with $n$ leaves is denoted by $\mathrm{PRT}_{n}$, and by PRT we denote the union $\bigcup_{n \geqslant 1} \mathrm{PRT}_{n}$. The number $n \geqslant 1$ of leaves will also be called the degree of the tree, and we also allow an empty tree $\emptyset$ of degree 0 later on (in Section 7). In low dimensions one gets:

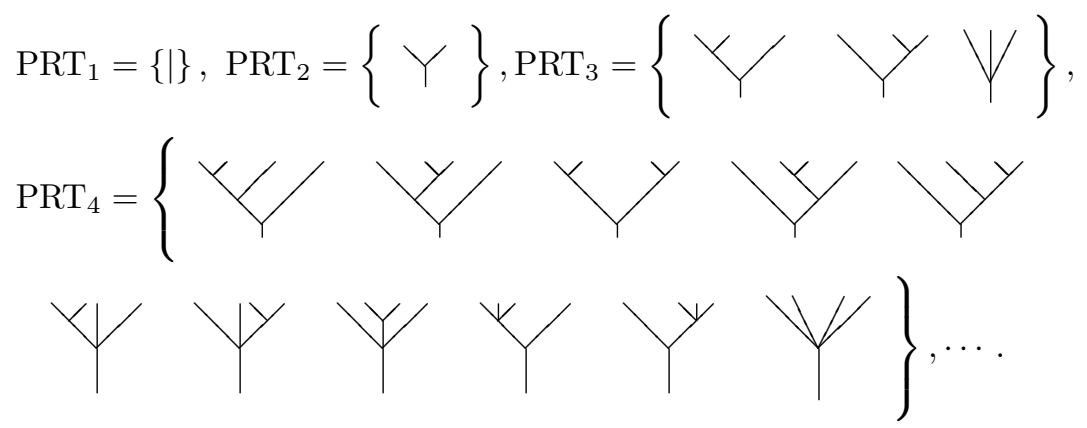

The $n$-grafting of $n$ trees is the gluing of the root of each tree on a new root. For example, the 2-grafting of the two trees $t$ and $s$ is:

$$
\vee_{2}(t, s):=Y^{s},
$$

and the 3 -grafting of three trees $t, s$ and $u$ is:

$$
\vee_{3}(t, s, u):=\psi^{t}{ }^{s} u
$$

Remark 2.3. From our definition of a non-empty planar tree, any $t \in \mathrm{PRT}_{n}, n \in \mathbb{N}_{\geqslant 2}$, is of the form

$$
t=\vee_{k}\left(t_{1}, \ldots, t_{k}\right)
$$

for uniquely determined $k$ and non-empty trees $t_{1}, \ldots, t_{k}$.

Let $V$ be a vector space. A labelled tree of degree $n, n \geqslant 1$, is a tree $t$ endowed with a labelling of all leaves by elements $v_{1}, \ldots, v_{n} \in V$. We denote such a labelled tree by 
$\left(t, v_{1}, \ldots, v_{n}\right)$, and represent it as follows:

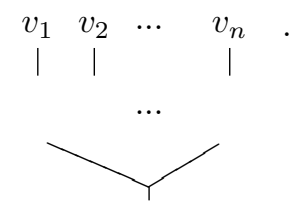

One defines the $n$-grafting of labelled trees by the $n$-grafting of the trees, where one keeps the labellings on the leaves.

We denote by $\mathrm{PRT}^{S}$ the subset $\mathrm{PRT}^{S} \subset \mathrm{PRT}$ that only contains planar reduced trees where the arities of all their inner vertices belong to $S \subset \mathbb{N}_{\geqslant 2}$. By $\mathrm{PRT}_{n}^{S}$ we denote the set of planar reduced trees in $\mathrm{PRT}^{S}$ with exactly $n$ leaves.

Example 2.4. Let $V$ be a vector space. The vector space $\oplus_{n \geqslant 1} \mathbb{K}\left[\mathrm{PRT}_{n}^{S}\right] \otimes V^{\otimes n}$, spanned by the labelled planar trees endowed with the $n$-grafting $\vee_{n}$ of labelled trees, for all $n \in S$, is an $S$-magmatic algebra.

Definition 2.5. Let $S \subset \mathbb{N}_{\geqslant 2}$ be a subset. An $S$-magmatic coalgebra $C$ is a vector space endowed with an $n$-ary co-operation $\Delta_{n}$ for each $n \in S$.

Given $t \in$ PRT a non-empty tree, it can be uniquely written as the $n$-grafting of non-empty trees $t_{1}, \ldots, t_{n}$. Then the $n$-ungrafting of planar trees is defined as follows:

$$
\wedge_{n}(t):=t_{1} \otimes \cdots \otimes t_{n}
$$

and the $m$-ungrafting $\wedge_{m}$ of such a tree is zero for $m \neq n$.

Example 2.6. Let $V$ be a vector space. The vector space $\oplus_{n \geqslant 1} \mathbb{K}\left[\mathrm{PRT}_{n}^{S}\right] \otimes V^{\otimes n}$, spanned by the labelled planar trees endowed with the $n$-ungrafting $\wedge_{n}$ of labelled trees, for all $n \in S$, is an $S$-magmatic coalgebra.

\section{Non-symmetric operads}

We recall some properties of non-symmetric operads. The operadic setting is quite useful for our purpose, and it will permit us to easily derive the combinatorics of the operads involved later on.

Definition 3.1. To a graded vector space

$$
M=\left(M_{0}, M_{1}, \ldots, M_{n}, \ldots\right),
$$

we associate its Schur functor $\widetilde{M}:$ Vect $\rightarrow$ Vect defined by

$$
\widetilde{M}(V):=\oplus_{n \geqslant 0} M_{n} \otimes_{\mathbb{K}} V^{\otimes n} .
$$

The Schur functor admits a tensor product and a composition defined by $\widetilde{M \otimes N}=$ $\widetilde{M} \otimes \widetilde{N}$ and $\widetilde{M \circ N}=\widetilde{M} \circ \widetilde{N}$. The tensor product and the composition of Schur functors can be seen as a Schur functor on graded vector spaces denoted by $M \otimes N$ and 
defined as:

$$
(M \otimes N)_{n}:=\bigoplus_{i+j=n} M(i) \otimes N(j),
$$

and their composite denoted by $M \circ N$ is defined as:

$$
(M \circ N)_{n}:=\bigoplus_{i_{1}+\cdots+i_{k}=n} M_{k} \otimes\left(N_{i_{1}} \otimes \cdots \otimes N_{i_{k}}\right) .
$$

Definition 3.2. A non-symmetric operad (often abbreviated into operad in this paper) is a graded vector space $\mathcal{P}=\left\{\mathcal{P}_{n}\right\}_{n \geqslant 0}$ equipped with composition maps

$$
\gamma_{i_{1}, \ldots, i_{n}}: \mathcal{P}_{n} \otimes \mathcal{P}_{i_{1}} \otimes \cdots \otimes \mathcal{P}_{i_{n}} \rightarrow \mathcal{P}_{i_{1}+\cdots+i_{n}}
$$

and an element Id $\in \mathcal{P}_{1}$, such that the transformations of functors $\gamma: \mathcal{P} \circ \mathcal{P} \rightarrow \mathcal{P}$ and $\iota:$ Id $\rightarrow \mathcal{P}$, deduced from this data, endows $(\mathcal{P}, \gamma, \iota)$ with a monoidal structure on the Schur functor $\mathcal{P}$.

We will only consider operads $\mathcal{P}$ with $\mathcal{P}_{0}=0, \mathcal{P}_{1}=\mathbb{K}$ Id (connected non-symmetric operads).

Let $F$ be the functor from the category of such operads to the category of graded vector spaces, which forgets the monoidal structure (and in particular deletes $\mathcal{P}_{1}$ ). Its left adjoint is the free non-symmetric operad functor,

$$
M=\left(0,0, M_{2}, M_{3}, \ldots, M_{n}, \ldots\right) \mapsto \mathcal{T}(M) .
$$

With PRT denoting the set of planar reduced trees, the arity $n$-component $\mathcal{T}(M)_{n}$ of the non-symmetric operad $\mathcal{T}(M)$ can be identified with the vector space

$$
\bigoplus_{t \in \mathrm{PRT}_{n}} M_{i_{1}} \otimes \cdots \otimes M_{i_{k}}
$$

where $k$ is the number of internal vertices of $t \in \mathrm{PRT}_{n}$ and $\left(i_{1}, \ldots, i_{k}\right)$ lists the arities of the internal vertices.

The operad composition can be described by the plugging (by some authors also called grafting) of trees onto the leaves (compare also Definition 3.5).

Definition 3.3. Let $\mathcal{P}$ be a non-symmetric operad (or an operad), and let $\mathcal{I}(n)$ be a subspace of $\mathcal{P}_{n}$ for each $n$.

Then $\mathcal{I}=\left(\mathcal{I}_{n}\right)_{n \in \mathbb{N} \geqslant 2}$ is called a (two-sided) ideal of $\mathcal{P}$, if for

$$
\gamma_{i_{1}, \ldots, i_{n}}\left(p \otimes q_{1} \otimes \ldots \otimes q_{n}\right)
$$

defined in $\mathcal{P}$, it follows that

$$
\gamma_{i_{1}, \ldots, i_{n}}\left(p \otimes q_{1} \otimes \ldots \otimes q_{n}\right) \in \mathcal{I}
$$

whenever at least one of the operations $p, q_{1}, \ldots, q_{n}$ is in $\mathcal{I}$.

The quotient of $\mathcal{P}$ by $\mathcal{I}$ is again a non-symmetric operad and it will be denoted by $\mathcal{P} / \mathcal{I}$.

As in rings, we analogously define right ideals in $\mathcal{P}, \mathcal{P}$ a non-symmetric operad. For $\mathcal{M}=\left(\mathcal{M}_{n}\right)_{n \in \mathbb{N} \geqslant 2}$ with $\mathcal{M}_{n} \subset \mathcal{P}_{n}$, the right ideal $\mathcal{I}:=\mathcal{M} \circ \mathcal{P}$ generated by $\mathcal{M}$ in $\mathcal{P}$ consists of all elements of the form $\gamma_{i_{1}, \ldots, i_{n}}\left(p \otimes q_{1} \otimes \ldots \otimes q_{m}\right)$ with $p \in \mathcal{M}_{n}$, $q_{1}, \ldots, q_{n} \in \mathcal{P}($ all $n)$. 
Definition 3.4. An algebra over a non-symmetric operad $\mathcal{P}$ (in short: $\mathcal{P}$-algebra) is a vector space $A$ equipped with a family of linear maps $\gamma_{A, n}: \mathcal{P}_{n} \otimes A^{\otimes n} \rightarrow A$, called structure maps. In particular, for $V$ a vector space, the underlying vector space of the free $\mathcal{P}$-algebra $\mathcal{P}(V)$ is associated to $V$ by the Schur functor $\mathcal{P}$, and the structure maps $\gamma_{A, n}$ combine to a linear map $\gamma_{A}: \mathcal{P}(A) \rightarrow A$, such that the following diagrams are commutative:
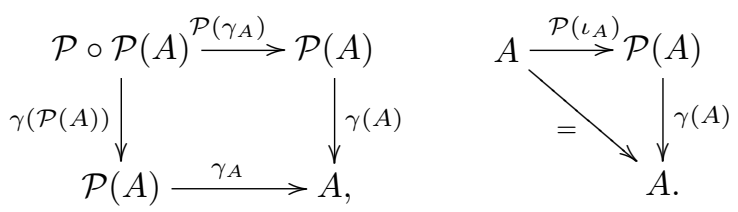

Morphisms of $\mathcal{P}$-algebras $A \rightarrow B$ are linear maps $\varphi: A \rightarrow B$ compatible with the corresponding structure maps $\gamma_{A}, \gamma_{B}$, such that the following diagram commutes:

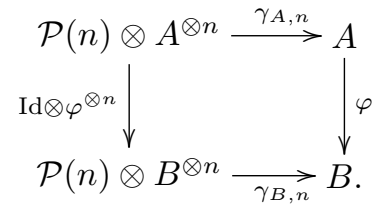

A $\mathcal{P}$-algebra $A$ is called nilpotent, if for sufficiently large $n$,

$$
\gamma_{A}(n)\left(\mu_{n} \otimes a_{1} \otimes \ldots a_{n}\right)=0
$$

for all $a_{1}, \ldots, a_{n} \in A, \mu_{n} \in \mathcal{P}_{n}$.

Analogously defined is the notion of a coalgebra over a non-symmetric operad; it is a vector space $C$ together with a family of structure maps $\lambda_{C, n}: \mathcal{P}_{n} \otimes C \rightarrow C^{\otimes n}$.

Morphisms of $\mathcal{P}$-coalgebras $C \rightarrow D$ are $\mathbb{K}$-linear maps $\varphi: C \rightarrow D$ compatible with the corresponding structure maps.

Following Loday $[\mathbf{9}]$, we define the notion of a connected coalgebra as follows. A $\mathcal{P}$-coalgebra $C$ is called connected or (co-)nilpotent, if the following condition holds:

For all $c \in C$, there exists $r \in \mathbb{N}$ such that for $n>r, \lambda_{C, n}\left(\delta_{n} \otimes c\right)=0$, for all $\delta_{n} \in \mathcal{P}_{n}$.

For any $\mathcal{P}$-coalgebra $C, r \geqslant 1$, we put

$$
F_{r} C:=\bigcap_{n>r} \bigcap_{\delta_{n} \in \mathcal{P}_{n}}\left\{x \in C \mid \delta_{n}(x)=0\right\} .
$$

$C$ is connected if and only if $\bigcup_{r \geqslant 1} F_{r} C=C$. Note that $\delta_{n} \in \mathcal{P}_{n}$ is any composition of the generating co-operations such that $\delta_{n}: C \rightarrow C^{\otimes n}$.

The $S$-magmatic algebra (Definition 2.1) and the $S$-magmatic coalgebra (Definition 2.5) can be viewed as algebras over the non-symmetric operad $M a g^{S}$, introduced as follows:

Definition 3.5. Let $S \subset \mathbb{N}_{\geqslant 2}$ be a set. Let $M$ be the graded vector space given by $M_{n}=\mathbb{K}$ for $n \in S$ and $M_{n}=0$ otherwise. Then $\operatorname{Mag}^{S}:=\mathcal{T}(M)$ is the free nonsymmetric operad generated by one $n$-ary generating operation for each $n \in S$. 
We can identify the space $M a g_{n}^{S}$ of operations ( $n$ inputs) with the vector space $\mathbb{K}\left[\mathrm{PRT}_{n}^{S}\right]$. The composition of operations corresponds to the plugging of trees onto leaves.

Thus we write $\operatorname{Mag}^{S}(V):=\oplus_{n} \mathrm{PRT}_{n}^{S} \otimes V^{\otimes n}$ for the (underlying vector space of the) free $S$-magmatic algebra.

Remark 3.6. Note that all non-symmetric operads $\mathrm{Mag}^{S}$ are included in $\mathrm{Mag}^{\infty}=$ $M a g^{\mathbb{N} \geqslant 2}$, also denoted by $\operatorname{Mag}^{\omega}$; see [7].

Moreover, for every inclusion $T \rightarrow S$ there is an inclusion of operads $\mathrm{Mag}^{T} \rightarrow \mathrm{Mag}^{S}$.

Definition 3.7. Let $\mathrm{Nil}^{S}$ denote the non-symmetric operad given by the quotient of $M a g^{S}$ with respect to the (two-sided) ideal consisting of all nontrivial compositions; i.e., any nontrivial composition will be zero in the quotient.

The following combinatorial formula is the key to make the dimensions of the spaces $\left(M a g^{S}\right)_{n}$ explicit (i.e. of the spaces of operations with $n$ inputs).

Proposition 3.8. For every $n \in \mathbb{N}_{\geqslant 2}$, the dimension $a_{n}^{S}:=\operatorname{dim}\left(\left(M a g^{S}\right)_{n}\right)$ is given by the following formula (in the ring of power series in one variable $x$ over $\mathbb{K}$ ):

$$
\left(x-\sum_{i \in S} x^{i}\right) \circ\left(\sum_{n \geqslant 1} a_{n}^{S} x^{n}\right)=x,
$$

where $\circ$ is the composition of power series.

Proof. For a presented quadratic non-symmetric operad $\mathcal{P}$, let $\mathcal{P}_{n}^{d}$ be the space spanned by the $n$-ary operations constructed out of $d$ generating operations, and let $f_{\mathcal{P}}(x, z)$ be defined as follows:

$$
f_{\mathcal{P}}(x, z):=\sum_{\substack{n \geqslant 1 \\ d \geqslant 0}} \operatorname{dim} \mathcal{P}_{n}^{d} z^{d} x^{n} .
$$

The operad $\mathrm{Mag}^{S}$ is free and especially Koszul. In the Koszul duality theory of operads, as noted for binary quadratic operads in [6], the Poincaré series of a Koszul operad and its Koszul dual are related.

Since we do not work with binary quadratic operads, but with general quadratic operads, we need the following general formula for Koszul operads, given by B. Vallette in $[\mathbf{1 7}$, Section 9]:

$$
f_{\mathcal{Q}}\left(f_{\mathcal{P}}(x, z),-z\right)=x \text {, where } \mathcal{P}^{!}=\mathcal{Q} \text { denotes the Koszul dual operad of } \mathcal{P} .
$$

In our case, the Koszul dual of $\mathrm{Mag}^{S}$ is exactly the operad $\mathrm{Nil}^{S}$.

We put $z=1$ and set $f(x):=f_{\left(M_{a g}\right)}(x, 1)=\sum_{n \geqslant 1} \operatorname{dim}\left(\left(M_{a g}\right)_{n}\right) x^{n}$.

We get the following Lagrange inversion type formula:

$$
g \circ f=\mathrm{Id}, \text { for } g(x)=\sum_{\substack{n \geqslant 1 \\ d \geqslant 0}}(-1)^{d} \operatorname{dim}\left(\left(N i l^{S}\right)_{n}^{d}\right) x^{n} .
$$

It is clear that the space $\left(N i l^{S}\right)_{1}^{0}=\mathbb{K}$ Id is one-dimensional. Also it is clear that $\operatorname{dim}\left(\left(N i l^{S}\right)_{n}^{1}\right)=1$ for all $n \in S$, while $\operatorname{dim}\left(\left(N i l^{S}\right)_{n}^{d}\right)=0$ for any other $n, d$.

Thus $f(x)$ is the composition inverse of $g(x)=x-\sum_{n \in S} x^{n}$.

We illustrate this formula in some particular cases in Section 8. 


\section{4. $(T ; S)$-magmatic bialgebras}

This section introduces the natural notion of $(T ; S)$-magmatic bialgebra that we will classify in Section 6 under the connectedness property.

The following proposition highlights that the vector space $\oplus_{n \geqslant 1} \mathbb{K}\left[\mathrm{PRT}_{n}^{S}\right]$ admits an algebra structure due to the $n$-grafting of trees, $\vee_{n}$ for $n \in S$, and a coalgebraic structure by endowing it with the $m$-ungrafting of the trees, $\wedge_{m}$ for $m \in S$.

Proposition 4.1. Let $V$ be a vector space.

(i) The space $\operatorname{Mag}^{S}(V)$ endowed with the $n$-grafting of labelled trees, $\vee_{n}$ for all $n \in S$, is the free $S$-magmatic algebra. Explicitly, for $i: V \rightarrow \operatorname{Mag}^{S}(V)$ the inclusion, the following universal property is fulfilled: Any linear map $f: V \rightarrow A$, where $A$ is any $S$-magmatic algebra, extends to a unique morphism of $S$-magmatic algebras $\tilde{f}: \operatorname{Mag}^{S}(V) \rightarrow A$ with $f=\tilde{f} \circ i$.

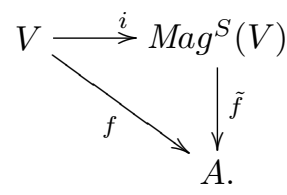

(ii) The space $\operatorname{Mag}^{S}(V)$ endowed with the $n$-ungrafting of labelled trees, $\wedge_{n}$ for all $n \in S$, is the cofree $S$-magmatic coalgebra. Explicitly, for $p: \operatorname{Mag}^{S}(V) \rightarrow V$ the projection on $V$, the following universal property is fulfilled: Any linear map $\phi: C \rightarrow V$, where $C$ is any connected $T$-magmatic coalgebra, extends to a unique coalgebra morphism $\tilde{\phi}: C \rightarrow \operatorname{Mag}^{S}(V)$ :

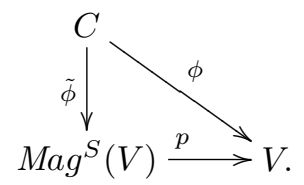

Assertions (i) and (ii) of the above proposition are dual to each other, and the proofs are by direct inspection; cf. similar proofs in $[\mathbf{3}]$.

As we have an algebraic and a coalgebraic structure on the same vector space, a natural question would be to seek relations between the operation and co-operations. Note that in the case of classical bialgebras, the relation (called the Hopf relation) between the operation and co-operation is determined by the fact that one is a homomorphism for the other.

Lemma 4.2. The relation between the $n$-grafting $\vee_{n}$ of trees and the $m$-ungrafting $\wedge_{m}$ of trees is the following: Let $t_{1}, \ldots, t_{n} \in \mathrm{PRT}^{S}$ be trees. Then

$$
\wedge_{m} \circ \vee_{n}\left(t_{1}, \ldots, t_{n}\right)= \begin{cases}0 & \text { if } m \neq n, \\ t_{1} \otimes \cdots \otimes t_{n} & \text { otherwise. }\end{cases}
$$

Proof. The ungrafting of trees is defined in Example 2.6. The tree

$$
t=\vee_{n}\left(t_{1} \otimes \cdots \otimes t_{n}\right)
$$

is uniquely decomposed as a product of $n$ trees, by construction of the space $\mathbb{K}\left[\mathrm{PRT}^{S}\right]$. 
By definition, the ungrafting depends only on the arity $n$ of the root. This ends the proof.

The above proposition motivates the following definition:

Definition 4.3. Let $T \subset S \subset \mathbb{N}_{\geqslant 2}$ be two sets.

A $(T ; S)$-magmatic bialgebra $\left(\mathcal{H}, \mu_{n}, \Delta_{n}\right)$ is a vector space $\mathcal{H}$ endowed with a $S$-magmatic algebra and a $T$-magmatic coalgebra structure verifying the following compatibility relation for all $k \in T$ and $l \in S$ :

$$
\begin{aligned}
& \Delta_{k} \circ \mu_{k}=\mathrm{Id} \text {, } \\
& \Delta_{k} \circ \mu_{l}=0 \quad \text { for } k \neq l \text {. }
\end{aligned}
$$

Remark 4.4. This is a generalisation of the $(\underline{m} ; \underline{n})$-magmatic bialgebra structure introduced in $[\mathbf{3}]$ in the non-unital framework. (The compatibility relations collapse to the ones above in the non-unital framework.)

Proposition 4.5. There exists a unique family of coproducts $\Delta_{m}$ with $m \in S$ such that $\Delta_{m}(v)=0$ for all $v \in V$ on the free $S$-magmatic algebra $M g^{S}(V)$, which makes it into an $(S ; S)$-magmatic bialgebra for which $V$ is primitive. Moreover, as a coalgebra $\operatorname{Mag}^{S}(V)$ is connected.

Proof. The existence is a consequence of Lemma 4.2.

The uniqueness is due to the compatibility relation. Indeed, let us construct

$$
\Delta_{m}: \operatorname{Mag}^{S}(V) \rightarrow \operatorname{Mag}^{S}(V)^{\otimes m},
$$

for all $m \in S$, as a $S$-magmatic coalgebra co-operation induced by $\Delta_{m}(v)=0$ and verifying the compatibility relation (1). As any tree $t \in \mathrm{PRT}^{S}$ can be uniquely viewed as the $n$-grafting of some trees $t_{1}, \ldots, t_{n}$, the $m$-ary co-operation on $t$ evaluates to

$$
\Delta_{m}(t)=\Delta_{m} \circ \vee_{n}\left(t_{1} \otimes \cdots \otimes t_{n}\right) .
$$

Therefore by the compatibility relation we get:

$$
\Delta_{m} \circ \vee_{n}\left(t_{1}, \ldots, t_{n}\right)= \begin{cases}0 & \text { if } m \neq n, \\ t_{1} \otimes \cdots \otimes t_{n} & \text { otherwise }\end{cases}
$$

This proves the uniqueness (there is no other choice to construct the $m$-ary cooperations).

The connectedness is proven in a similar way as in $[\mathbf{3}]$ and gives the following filtration:

$$
F_{r}\left(\operatorname{Mag}^{S}(V)\right)=\oplus_{n=1}^{r} \mathbb{K}\left[\mathrm{PRT}_{n}^{S}\right] \otimes V^{\otimes n}
$$

and Prim $\operatorname{Mag}^{S}(V)=V$. (Note that we are in the case of a $(S ; S)$-magmatic bialgebra and not in the case of $(T ; S)$-magmatic bialgebra where $T \neq S$.)

Note that the constructed co-operation $\Delta_{n}$ is exactly $\wedge_{n}$ by uniqueness. 


\section{The $S_{T}$-rooted magmatic algebra}

In the following, we describe a non-symmetric operad related to the $S$-magmatic operad $M a g^{S}$ and the $T$-magmatic operad $M a g^{T}$. It will occur in the structure theorems.

Definition 5.1. Let $\mathcal{I}=\left(\mathcal{I}_{n}\right)_{n \in \mathbb{N} \geqslant 2}$ be the right ideal generated by the $k$-ary operations $\mu_{k}$ for all $k \in S \backslash T$ in the non-symmetric operad $M^{S} g^{S}$ (see Definition 3.3). We set $\left(M a g_{\text {root } \backslash T}^{S}\right)_{1}:=\mathbb{K}$ Id and $\left(\operatorname{Mag}_{\text {root } \backslash T}^{S}\right)_{n}:=\mathcal{I}_{n}$ for $n>1$.

Proposition 5.2. We get a non-symmmetric operad

$$
M a g_{\text {root } \backslash T}^{S}=\left(\left(M_{\text {root } \backslash T}^{S}\right)_{n}\right)_{n \in \mathbb{N} \geqslant 2} .
$$

Moreover, the $S_{T}$-rooted magmatic operad is freely generated by operations

$$
\mu_{k} \circ\left(\nu_{1}^{S} \otimes \cdots \otimes \nu_{k}^{S}\right)
$$

where $k \in S \backslash T$ and $\nu_{i}^{S}$ are operations that are compositions of generating operations from the $S$-magmatic operad but they do not belong to the $S_{T}$-rooted magmatic operad. The operad structure is given by its composition map

$$
\gamma: M a g_{\text {root } \backslash T}^{S} \circ M a g_{\text {root } \backslash T}^{S} \rightarrow M a g_{\text {root } \backslash T}^{S},
$$

which is the composition of operations and its unit map

$$
\iota: \mathrm{Id} \rightarrow M a g_{\text {root } \backslash T}^{S},
$$

which is given by the operation identity. It is a sub-operad of the S-magmatic operad.

Proof. It is easy to verify the monoidal axioms. Moreover, there is a unique way of writing the operations as a composition of generating operations (this gives the injectivity of the map $S_{T}$-rooted magmatic operad to $S$-magmatic operad).

Definition 5.3. A $S_{T}$-rooted magmatic algebra $A$ is an algebra over the free operad $M a g_{\text {root } \backslash T}^{S}$ (i.e. it is a vector space endowed with operations $\mu_{k}$, indexed by $S \backslash T$, and $\mu_{k} \circ\left(\nu_{1}^{S} \otimes \cdots \otimes \nu_{k}^{S}\right)$ as above $)$.

We will denote by $\mathrm{PRT}_{r o o t \backslash T}^{S}$ the set of trees $t \in \mathrm{PRT}^{S}$ such that the arity of the root is in $S \backslash T$. By $\left(\operatorname{PRT}_{\operatorname{root} \backslash T}^{S}\right)_{n}$ we denote the subset of trees having exactly $n$ leaves.

We can identify the space $\left(M a g_{\text {root } \backslash T}^{S}\right)_{n}$ of operations ( $n$ inputs) with the vector space $\mathbb{K}\left[\left(\mathrm{PRT}_{\operatorname{root} \backslash T}^{S}\right)_{n}\right]$. The composition of operations corresponds to the plugging of trees onto leaves.

Thus we write $M a g_{\text {root } \backslash T}^{S}(V):=\oplus_{n} \mathbb{K}\left[\left(\mathrm{PRT}_{\text {root } \backslash T}^{S}\right)_{n}\right] \otimes V^{\otimes n}$ for the underlying vector space of the free $S_{T}$-rooted magmatic algebra.

Example 5.4. To illustrate the above identification, we consider the operation $\mu_{2} \circ\left(\operatorname{Id} \otimes \mu_{3}\right) \circ\left(\operatorname{Id} \otimes \operatorname{Id} \otimes \operatorname{Id} \otimes \mu_{3}\right)$, in the space of operations of $\left(\operatorname{Mag} g_{\text {root } \backslash\{3,4\}}^{\{2,3,4\}}\right)_{6}$,

which can be depicted as the following tree:

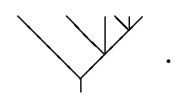


One key part of the proof of the structure theorem is the construction of the forgetful functor from the algebra structure of the bialgebra to its primitive part. The universal enveloping functor is the left adjoint functor to this forgetful functor and is constructed quite naturally.

Let $T \subset S \subset \mathbb{N}_{\geqslant 2}$ be two sets. Let $A$ be a $S$-magmatic algebra. By definition, it is equipped with operations $\mu_{n}$, and consequently, with all possible compositions of them. By

$$
\left.\mu_{k} \circ \text { (composition of operations indexed by } \mathrm{S}\right) \text {, }
$$

where $k$ runs through all elements of $S \backslash T$, we construct an infinite family of operations (contained in the family of all operations).

Proposition 5.5. The above construction defines a functor

$$
()_{M_{\text {rag }}^{S} \backslash \backslash T}^{S}:\{S \text {-magmatic algebra }\} \rightarrow\left\{S_{T} \text {-rooted magmatic algebra }\right\}
$$

$\left.\left(A,\left\{\mu_{k}\right\}_{k \in S}\right) \mapsto\left(A,\left\{\mu_{k} \circ \text { (composition of operations indexed by } S\right)\right\}_{k \in S \backslash T}\right)$,

namely the forgetful functor.

Proof. Definition 5.3 ensures that the constructed algebra

$$
\left.\left(A,\left\{\mu_{k} \circ \text { (composition of operations indexed by } \mathrm{S}\right)\right\}_{k \in S \backslash T}\right)
$$

is a $S_{T}$-rooted magmatic algebra.

We construct a functor

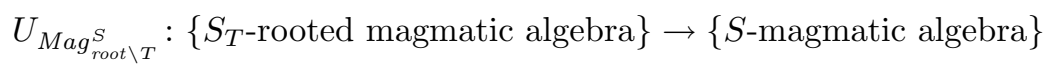

as follows: For any $S_{T}$-rooted magmatic algebra $\left(A,\left\{\tilde{\mu}_{k} \circ\left(\tilde{\nu}_{1}^{S} \otimes \cdots \otimes \tilde{\nu}_{k}^{S}\right)_{k \in S \backslash T}\right\}\right)$, let the $S$-magmatic algebra $U_{M a g_{\text {root } \backslash T}^{S}}(A)$ be constructed as the free $S$-magmatic algebra over the vector space $A$ quotiented by the relations which identify

$$
\tilde{\mu}_{k} \circ\left(\tilde{\nu}_{1}^{S} \otimes \cdots \otimes \tilde{\nu}_{k}^{S}\right), \quad \text { for } k \in S \backslash T,
$$

with the corresponding operations $\mu_{k} \circ\left(\nu_{1}^{S} \otimes \cdots \otimes \nu_{k}^{S}\right)$ in the free $S$-magmatic algebra.

Proposition 5.6. The universal enveloping functor

$$
U_{M a g_{\text {root } \backslash T}^{S}}:\left\{S_{T} \text {-rooted magmatic algebra }\right\} \rightarrow\{S \text {-magmatic algebra }\}
$$

is left adjoint to the forgetful functor

$$
()_{M_{\text {root } \backslash T}^{S}}^{S}:\{S \text {-magmatic algebra }\} \rightarrow\left\{S_{T} \text {-rooted magmatic algebra }\right\} .
$$

Proof. Let $A$ be a $S$-magmatic algebra and $B$ be a $S_{T}$-rooted magmatic algebra. On the one hand, let $f: B \rightarrow(A)_{M a g_{\text {root } \backslash T}^{S}}$ be a $S_{T}$-rooted magmatic algebra morphism. It determines uniquely a morphism of $S$-magmatic algebra $\operatorname{Mag}^{S}(B) \rightarrow A$ since $A$ is a $S$-magmatic algebra and $M a g^{S}$ is the free $S$-magmatic algebra.

On the other hand, let $g: U_{M a g_{\text {root } \backslash T}^{S}}(B) \rightarrow A$ be a $S$-magmatic algebra morphism. The construction of the universal enveloping functor $U_{M a g_{\text {root } \backslash T}^{S}}$ shows that the map 
$B \rightarrow U_{M a g_{\text {root } \backslash T}^{S}}(B)$ is a $S_{T}$-rooted magmatic algebra morphism. Hence the composition with $g$ gives a $S_{T}$-rooted magmatic algebra morphism $B \rightarrow(A)_{M_{\text {rag }}^{S} \backslash T}$.

Corollary 5.7. The universal enveloping functor of the free $S_{T}$-rooted magmatic algebra is isomorphic to the free $S$-magmatic algebra:

$$
U_{M a g_{\text {root } \backslash T}^{S}}\left(\operatorname{Mag}_{\text {root } \backslash T}^{S}(V)\right) \cong \operatorname{Mag}^{S}(V) .
$$

Proof. Note that the underlying vector space is preserved under the forgetful functor ()$_{M a g_{\text {root } \backslash T}^{S}}$. As the universal enveloping functor $U_{M a g_{\text {root } \backslash T}^{S}}$ is left adjoint to the functor ()$_{M_{\text {root } \backslash T}^{S}}^{S}$, and the left adjoint of the forgetful functor

$$
\left\{S_{T} \text {-rooted magmatic algebra }\right\} \rightarrow \text { Vect }
$$

is the free functor, their composite is left adjoint to the forgetful functor

$$
\{S \text {-magmatic algebra }\} \rightarrow \text { Vect. }
$$

Therefore it is the free functor Vect $\rightarrow\{S$-magmatic algebra $\}$.

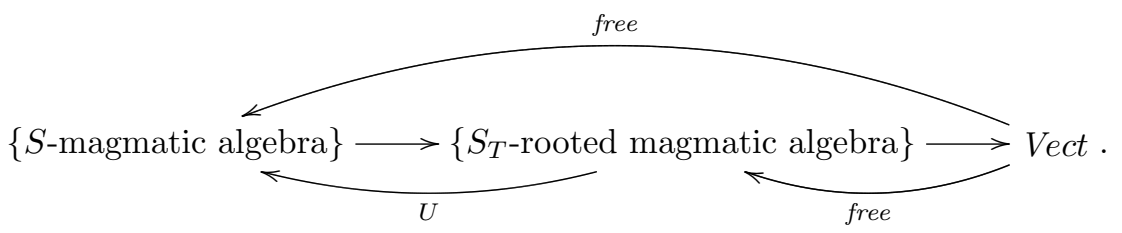

Corollary 5.8. For any $S_{T}$-rooted magmatic algebra $A, U_{M a g_{\text {root } \backslash T}^{S}}(A)$ is a connected $(S ; S)$-magmatic bialgebra.

Proof. It is a consequence of the above Corollary 5.7 and Proposition 4.5.

\section{Main theorem}

In this section, we state the classification theorem for connected $(T ; S)$-magmatic bialgebras which we call the structure theorem. In order to prove it, we focus on the construction of the algebra of the primitive elements, and on the rigidity theorem in the particular case of an $(S ; S)$-magmatic bialgebra.

Definition 6.1. Let $C$ be a $T$-magmatic coalgebra. The vector space of primitive elements Prim $C$ is defined as

$$
\text { Prim } C:=\cap_{n \in T}\left\{x \in \mathcal{H} \mid \Delta_{n}(x)=0\right\} .
$$

The following theorem gives the classification of the connected $(T ; S)$-magmatic bialgebras. 
Theorem 6.2. Let $T \subset S \subset \mathbb{N}_{\geqslant 2}$ be two sets. If $\mathcal{H}$ is a $(T ; S)$-magmatic bialgebra over a field $\mathbb{K}$, then the following are equivalent:

(i) $\mathcal{H}$ is a connected $(T ; S)$-magmatic bialgebra.

(ii) $\mathcal{H}$ is isomorphic to $U_{M a g_{\text {root } \backslash T}^{S}}(\operatorname{Prim} \mathcal{H})$ as a $(T ; S)$-magmatic bialgebra.

(iii) $\mathcal{H}$ is cofree among the connected T-magmatic coalgebra.

To prove the above theorem, we have to make the primitive part of a $T$-magmatic coalgebra explicit.

Proposition 6.3. The primitive part of a $(T ; S)$-magmatic bialgebra is generated by the operations $\mu_{k}$ and all its composites $\mu_{k} \circ\left(\nu_{1} \otimes \cdots \otimes \nu_{k}\right)$ for $k \in S \backslash T$, and $\nu_{i}$ are operations generated by operations indexed by $S$.

Proof. By definition, the compatibility relation (1) gives $\Delta_{k} \circ \mu_{l}=0$ for any $k \neq l$. It comes naturally that $\mu_{k}$, for $k \in S \backslash T$, is primitive. Indeed, first we have $\Delta_{m} \circ \mu_{k}=0$ for all $m \in T$; therefore any composite of the generating co-operations $\delta$ composed with $\mu_{k}$ will be zero. This gives the primitiveness property. This is also true for all its composites $\mu_{k} \circ\left(\mu_{i_{1}} \otimes \cdots \otimes \mu_{i_{k}}\right)$ such that $k \in S \backslash T$ and $\mu_{i_{1}}, \ldots, \mu_{i_{k}}$ composition of operations indexed by $S$.

As the $S$-magmatic operad is free, any operation can be uniquely written as a composition of the generating operations. Therefore there is a unique way to write an operation as $\mu_{k} \circ\left(\mu_{i_{1}} \otimes \cdots \otimes \mu_{i_{k}}\right)$, where $\mu_{k}$ is a generating operation and $\mu_{i_{j}}$ are operations of the $S$-magmatic operad. Suppose that $k \in T$. Then the $k$-ary co-operation $\Delta_{k}$ composed with $\mu_{k} \circ\left(\mu_{i_{1}} \otimes \cdots \otimes \mu_{i_{k}}\right)$ is not zero but equals $\mu_{i_{1}} \otimes \cdots \otimes \mu_{i_{k}}$, and $\mu_{k} \circ\left(\mu_{i_{1}} \otimes \cdots \otimes \mu_{i_{k}}\right)$ is not primitive in this case.

Therefore the only primitive operations are the operations $\mu_{k}$ and all its composites $\mu_{k} \circ\left(\mu_{i_{1}} \otimes \cdots \otimes \mu_{i_{k}}\right)$ such that $k \in S \backslash T$ and $i_{1}, \ldots, i_{k} \in S$, which ends the proof.

Example 6.4. We consider $(\{2,4\} ;\{2,3,4,5\})$-magmatic bialgebras, and especially the free algebra. Thus the tree $t:=\bigvee$ represents an element which is primitive. Indeed, the following holds:

$$
\delta_{2}(t)=\delta_{4}(t)=0,
$$

by the compatibility relations (1).

Proposition 6.5. Let $\left(\mathcal{H}, \mu_{n}, \Delta_{m}\right)$ be a $(T ; S)$-magmatic bialgebra. Its primitive part admits a $S_{T}$-rooted magmatic algebra structure.

Proof. The generating operations of the primitive part verify the conditions of Definition 5.3. 
Corollary 6.6. The primitive part of the free $S$-magmatic algebra $\operatorname{Mag}^{S}(V)$ over a vector space $V$ is exactly $\operatorname{Mag}_{\text {root } \backslash T}^{S}(V)$.

Proof. The first remark is that any tree $t$ which admits a root of arity $n \in S \backslash T$ is primitive. This is due to the fact that the tree can be uniquely rewritten as

$$
\mu_{n}\left(t_{1} \otimes \cdots \otimes t_{n}\right)
$$

for certain non-empty trees $t_{1}, \ldots, t_{n}$, and by the compatibility relation, gives the relation $\wedge_{m} \circ \vee_{n}=0$ (Lemma 4.2). This prove that these trees are primitive elements. Similarly, trees such that their root is of arity $n \in T$ are not primitive as there exists $\wedge_{n}$ such that $\wedge \circ \mu_{n}=\mathrm{Id}$. By the isomorphism identifying the trees with root of arity $n \in S \backslash T$ with $S_{T}$-rooted magmatic algebra, the proof is completed (Def. 5.3).

Now we focus on the particular case of the structure theorem where the sets $T$ and $S$ are equal. The rigidity theorem is a classification of connected $(S ; S)$-magmatic bialgebra where the primitive part admits a structure of vector space. The results of $[\mathbf{3}]$, where $S=\{2, \ldots, n\}$, can be extended to the context of $(S ; S)$-magmatic bialgebra with very few changes.

Definition 6.7. The completed $S$-magmatic algebra, denoted by $M a g^{S}(\mathbb{K})^{\wedge}$, is defined by

$$
\operatorname{Mag}^{S}(\mathbb{K})^{\wedge}=\prod_{n \geqslant 0} \operatorname{Mag}_{n}^{S}
$$

This definition allows us to consider formal power series of trees in $\operatorname{Mag}^{S}(\mathbb{K})^{\wedge}$; i.e. we consider formal power series in the non-associative variable, $\mid$, where $\mid$ denotes the generator of the one-dimensional space $\mathbb{K}=\operatorname{Mag}_{1}^{S}(\mathbb{K})$; cf. [5].

Definition 6.8. Let $\mathcal{H}$ be an $(S ; S)$-magmatic bialgebra, $n \in S$, and let $f_{1}, \ldots, f_{n}$ be linear maps $\mathcal{H} \rightarrow \mathcal{H}$. The $n$-convolution of $f_{1}, \ldots, f_{n}$ is the linear map defined by:

$$
\star_{n}\left(f_{1} \cdots f_{n}\right):=\mu_{n} \circ\left(f_{1} \otimes \cdots \otimes f_{n}\right) \circ \Delta_{n} .
$$

We define a map $\chi$ from the set of trees to vector space of operations of the $(S ; S)$ magmatic bialgebra: to the $n$-th corolla $t_{n}=\vee(\underbrace{|, \ldots,|}_{n})$ we associate the operation $\star_{n}$. As any other tree can be seen as a grafting of corollas of degree $k$, it is associated with the composition of the respective operations $\star_{k}$. We denote $\star_{t}:=\chi(t)$.

Theorem 6.9. Any connected $(S ; S)$-magmatic bialgebra $\mathcal{H}$ is isomorphic to

$$
\operatorname{Mag}^{S}(\operatorname{Prim} \mathcal{H}):=\left(\operatorname{Mag}^{S}(\operatorname{Prim} \mathcal{H}), \vee_{k}, \wedge_{k}\right)_{k \in S},
$$

where $\vee_{k}$ is $k$-grafting and $\wedge_{k}$ is the $k$-ungrafting.

Proof. Since the proof is similar to the proof of the analogous result for $m$-magmatic bialgebras in [3], we give only a sketch of the proof. 
Suppose that $\mathcal{H}$ is connected; we prove the isomorphism $\mathcal{H} \cong \operatorname{Mag}^{S}$ (Prim $\left.\mathcal{H}\right)$ by explicitly giving the two inverse maps. We define the $S$-magmatic coalgebra morphism: $G: \mathcal{H} \rightarrow \operatorname{Mag}^{S}$ (Prim $\mathcal{H}$ ) as the unique extension of the following linear map:

$$
x \mapsto x-\sum_{n \in S} \star_{n} \circ \mathrm{Id}^{\otimes n}(x) .
$$

Note that $e: \mathcal{H} \rightarrow \mathcal{H}$ defined as

$$
e:=\mathrm{Id}-\sum_{n \in S} \star_{n} \circ \mathrm{Id}^{\otimes n}
$$

plays the role of a projector on the primitive part.

Then we define the $S$-magmatic algebra morphism $F: \operatorname{Mag}^{S}(\operatorname{Prim} \mathcal{H}) \rightarrow \mathcal{H}$ as the unique extension of the linear map:

$$
x \mapsto \sum \star_{t}(\mathrm{Id})(x),
$$

where the sum extends on all non-empty planar trees $t \in \oplus_{n \geqslant 1} \mathbb{K}\left[\mathrm{PRT}_{n}^{S}\right]$.

Moreover, denote by $y$ the generator of $\operatorname{Mag}^{S}(\mathbb{K}), y:=\mid$, and by $y^{n}:=\vee_{n} \circ y^{\otimes n}$. We define $g(y):=y-\sum_{n \in S} y^{n}$, and $f(y):=\sum t$, where the sum extends on all planar trees $t \in \bigcup_{n \geqslant 1} \mathrm{PRT}_{n}^{S}$. Using that these two preceding maps are inverse with respect to the composition of such power series one can show that:

$$
F \circ G=\operatorname{Id}_{\mathcal{H}}, \quad G \circ F=\operatorname{Id}_{M a g^{S}}(\operatorname{Prim} \mathcal{H}) .
$$

Remark that $\mathcal{H} \cong \operatorname{Mag}^{S}($ Prim $\mathcal{H})$ is a $(S ; S)$-magmatic bialgebra. Indeed, we have the two following properties:

$$
\begin{aligned}
\vee_{n}\left(G\left(x_{1}\right), \ldots, G\left(x_{n}\right)\right) & =G \circ F\left(\vee_{n}\left(G\left(x_{1}\right), \ldots, G\left(x_{n}\right)\right)\right) \\
& =G \circ \mu_{n}\left(F \circ G\left(x_{1}\right), \ldots, F \circ G\left(x_{n}\right)\right) \\
& =G \circ \mu_{n}\left(x_{1}, \ldots, x_{n}\right) \\
\Delta_{n}(F(x)) & =((F \circ G) \otimes \cdots \otimes(F \circ G)) \circ \Delta_{n}(F(x)) \\
& =(F \otimes \cdots \otimes F) \circ \wedge_{n}(G \circ F(x)) \\
& =(F \otimes \cdots \otimes F) \circ \wedge_{n}(x),
\end{aligned}
$$

which proves that $F$ is moreover a $S$-magmatic coalgebra morphism (resp. $G$ is a $S$ magmatic algebra morphism and hence an $(S ; S)$-magmatic bialgebra morphism.

Now we can prove the structure theorem stated at the beginning of the section.

Before starting the proof we recall that the primitive space only depends on the co-operations (there is no unit here), i.e. on the coalgebraic structure.

Proof of Theorem 6.2. We prove the following implications:

(i) $\Rightarrow$ (iii). If $\mathcal{H}$ is a $(T ; S)$-magmatic bialgebra, then, by Theorem $6.9 \mathcal{H}$ is isomorphic to $\operatorname{Mag}^{T}(\operatorname{Prim} \mathcal{H})$ as a $(T ; T)$-magmatic bialgebra. Therefore $\mathcal{H}$ is cofree.

(iii) $\Rightarrow$ (ii). If $\mathcal{H}$ is cofree, then it is isomorphic to $\operatorname{Mag}^{T}$ (Prim $\left.\mathcal{H}\right)$ and Prim $\mathcal{H}$ is a $S_{T}$-rooted magmatic algebra. Moreover, $\operatorname{Mag}^{T}($ Prim $\mathcal{H}$ ) is a $S$-magmatic algebra by endowing the vector space $\operatorname{Mag}^{T}(\operatorname{Prim} \mathcal{H})$ with the products $\mu_{n}$ inherited by 
the products on $\mathcal{H}$. By adjunction (Proposition 5.6), the inclusion map Prim $\mathcal{H} \rightarrow$ $\operatorname{Mag}^{T}($ Prim $\mathcal{H})$ gives rise to a $S$-magmatic algebra morphism:

$$
\Phi: U_{\text {Mag }_{\text {root } \backslash T}^{S}}(\operatorname{Prim} \mathcal{H}) \rightarrow \operatorname{Mag}^{T}(\operatorname{Prim} \mathcal{H}) .
$$

On the other hand, the inclusion Prim $\mathcal{H} \rightarrow U_{M a g_{\text {root } \backslash T}^{S}}($ Prim $\mathcal{H})$ admits a unique extension

$$
\Psi: \operatorname{Mag}^{T}(\operatorname{Prim} \mathcal{H}) \rightarrow U_{\text {Mag }_{\text {root } \backslash T}^{S}}(\operatorname{Prim} \mathcal{H})
$$

Check that both maps are inverse one to the other.

(ii) $\Rightarrow(\mathrm{i})$. This is Corollary 5.8 .

Corollary 6.10. The following holds for the particular $(T ; S)$-magmatic bialgebra $\left(\operatorname{Mag}^{S}(V), \vee_{n}, \wedge_{m}\right)_{n \in S, m \in T}$ :

$$
\operatorname{Mag}^{S}(V)=\operatorname{Mag}^{T} \circ \operatorname{Mag}_{\text {root } \backslash T}^{S}(V) .
$$

Proof. The free $S$-magmatic algebra $\operatorname{Mag}^{S}(V)$ over a vector space $V$ is connected as a $S$-magmatic coalgebra (Proposition 4.5 ). Similarly it can be proven that it is connected as a $T$-magmatic coalgebra. Indeed, the filtration induced by the connectedness is:

$$
F_{r} \operatorname{Mag}_{\text {root } \backslash T}^{S}(V)=\bigoplus_{l=1}^{r} \mathbb{K}\left[\left(\mathrm{PRT}_{\text {root } \backslash T}^{S}\right)_{l}\right] \otimes V^{\otimes l}
$$

The proof is done by contradiction and descending induction as in $[\mathbf{3}]$.

Then applying the structure theorem 6.2 , we get that

$$
\operatorname{Mag}^{S}(V)=\operatorname{Mag}^{T}\left(\operatorname{Prim} \operatorname{Mag}^{S}(V)\right) .
$$

Corollary 6.6 ends the proof.

\section{The unital version of the $m, n$-magmatic bialgebra}

In this section, we treat a particular case which can be extended to the unital framework: $S=\{2, \ldots, n\}, T=\{2, \ldots, m\}$. First we have to redefine the notions of $\underline{n}$-magmatic algebra, $\underline{m}$-magmatic coalgebra, $(\underline{m} ; \underline{n})$-magmatic bialgebra in the unital framework. In this case, the compatibility relation between the operations and cooperations is different, but most of the conclusions of the non-unital framework are preserved.

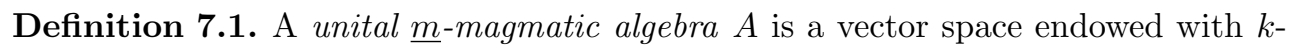
ary operations $\mu_{k}$, one for each $k$ with $2 \leqslant k \leqslant m$, which are unitary. That is, there exists a unit map $\eta: \mathbb{K} \rightarrow A, a \mapsto a \cdot 1$, such that

$$
\mu_{k}\left(x_{1}, \ldots, x_{k}\right)=\mu_{k-1}\left(x_{1}, \ldots, x_{i-1}, x_{i+1}, \ldots, x_{k}\right), \quad \text { where } x_{i}=1 \text { and } x_{j} \in A, \forall j \text {. }
$$


Diagrammatically this condition is the commutativity of:

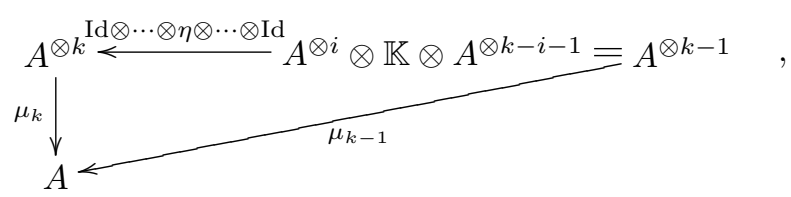

where $\eta: \mathbb{K} \rightarrow A$ is the unit map.

From now on, we denote by $\operatorname{Mag} \underline{\underline{m}}(V)$ the free unital $\underline{m}$-magmatic algebra on $V$.

Definition 7.2. A vector space $C=\mathbb{K} 1 \oplus \bar{C}$ is called a unital $\underline{m}$-magmatic coalgebra $C$ if it is endowed with $k$-ary co-operations $\Delta_{k}: C \rightarrow C^{\otimes k}$, one for each $2 \leqslant k \leqslant m$, which are co-unitary; i.e., the following diagram is commutative:

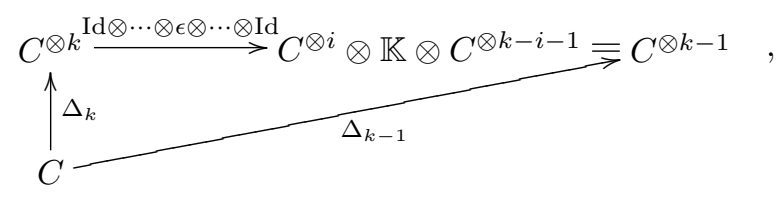

where $\epsilon: C \longrightarrow \mathbb{K}$ is the augmentation (and $\Delta_{1}:=\mathrm{Id}$ ).

Definition 7.3. Let $C=\mathbb{K} 1 \oplus \bar{C}$ be a unital $\underline{m}$-magmatic coalgebra. The set of $(p, q)$-shuffles is denoted $\operatorname{Sh}(p, q)$. It is the set of permutations of

$$
(1, \ldots, p, p+1, \ldots p+q)
$$

such that the image of the elements 1 to $p$ and, of the elements $p+1$ to $p+q$, are in increasing order. The reduced co-operations $\delta_{k}$ for $k \in \underline{m}$ are defined as follows:

$$
\begin{aligned}
& \delta_{1}(x):=x \\
& \delta_{k}(x):=\Delta_{k}(x)-\sum_{l \leqslant k-1} \sum_{\sigma \in \operatorname{Sh}(l, k-l)} \sigma \circ\left(\delta_{l}(x), 1^{\otimes k-l}\right) .
\end{aligned}
$$

Definition 7.4. Let $C$ be a unital $\underline{m}$-magmatic coalgebra. The vector space of primitive elements Prim $C$ is defined as

$$
\cap_{1<k \leqslant m}\left\{x \in C \mid \delta_{k}(x)=0 \text { for any } k \text {-ary reduced co-operation } \delta_{k}: C \rightarrow C^{\otimes k}\right\} .
$$

Moreover, $C$ is said to be connected if it verifies:

$$
C=\cup_{r} F_{r} C
$$

with

$$
F_{0} C=\mathbb{K}, F_{r} C:=\bigcap_{k>r}\left\{x \in C \mid \delta_{k}(x)=0 \text { for any } k \text {-ary reduced co-operation }\right\} .
$$

Example 7.5 (cf. [3]). The vector space of planar trees $\oplus_{l \geqslant 0} \mathbb{K}\left[\left(\mathrm{PRT}^{n}\right)_{l}\right]$, where $\mathrm{PRT}_{0}:=\{\emptyset\}$, can be endowed with an $\underline{n}$-magmatic algebra structure and an $\underline{n}$-magmatic coalgebra structure with the grafting and the ungrafting of trees defined 
as follows:

$$
\wedge_{k}(t):=\sum t_{1} \otimes \cdots \otimes t_{k}
$$

where the sum extends over all the ways to write $t$ as $\vee_{k}\left(t_{1}, \ldots, t_{k}\right)$, where $t_{i} \in \bigcup_{l \geqslant 0} \mathrm{PRT}_{l}$. This can be made explicit as follows: as for the tree $t$ there is a unique way to be written as a grafting $t=\vee_{k}\left(t_{1}, \ldots, t_{k}\right)$, where $t_{i} \neq \emptyset$ for all $i$ :

$$
\begin{aligned}
& \wedge_{k}(t):=\left(\begin{array}{cc}
t_{1} & \otimes \\
\left.\mid \begin{array}{l}
t_{k} \\
\wedge_{l}(t)
\end{array}\right)= \begin{cases}\sum_{i=0}^{l-1} \emptyset^{\otimes i} \otimes t \otimes \emptyset^{\otimes l-i-1}, & \sum_{i=0}^{k-1} \emptyset^{\otimes i} \otimes t \otimes \emptyset^{\otimes k-i-1}, \\
\sum_{i=0}^{l-1} \emptyset^{\otimes i} \otimes t \otimes \emptyset^{\otimes l-i-1} & \text { if } l<k \\
+\sum_{i_{1}+\cdots+i_{k+1}=l-k} \emptyset^{\otimes i_{1}} \otimes t_{1} \otimes \emptyset^{\otimes i_{2}} \otimes \cdots \otimes t_{k} \otimes \emptyset^{\otimes i_{k+1}}, & \text { if } l>k\end{cases} \\
\wedge_{l}(\mid):=\sum_{i=0}^{l-1} \emptyset^{\otimes i} \otimes \mid \otimes \emptyset^{l-i-1},
\end{array}\right. \\
& \wedge_{l}(\emptyset):=\emptyset^{\otimes l} .
\end{aligned}
$$

Definition 7.6. A unital $(\underline{m} ; \underline{n})$-magmatic bialgebra $\left(\mathcal{H}, \mu_{k}, \Delta_{l}\right)$, where $2 \leqslant k \leqslant m$, and $2 \leqslant l \leqslant m$ is a vector space $\mathcal{H}=\overline{\mathcal{H}} \oplus \mathbb{K} 1$ such that:

1. $\mathcal{H}$ admits a $\underline{n}$-magmatic algebra structure with $l$-ary operations denoted $\mu_{l}$,

2. $\mathcal{H}$ admits a $\underline{m}$-magmatic coalgebra structure with $k$-ary co-operations denoted $\Delta_{k}$

3. $\mathcal{H}$ satisfies the following "compatibility relation" for $2 \leqslant k \leqslant m$ and $2 \leqslant l \leqslant n$, and for all $x_{1}, \ldots, x_{l} \in \overline{\mathcal{H}}$ :

$$
\begin{aligned}
& \Delta_{l} \circ \mu_{l}\left(x_{1} \otimes \cdots \otimes x_{l}\right)=x_{1} \otimes \cdots \otimes x_{l}+\sum_{i=0}^{l-1} 1^{\otimes i} \otimes \underline{x} \otimes 1^{\otimes l-i-1}, \\
& \Delta_{k} \circ \mu_{l}\left(x_{1} \otimes \cdots \otimes x_{l}\right)= \\
& \begin{cases}\sum_{i=0}^{k-1} 1^{\otimes i} \otimes \underline{x} \otimes 1^{\otimes k-i-1}, & \text { if } k<l \\
\sum_{i=0}^{k-1} 1^{\otimes i} \otimes \underline{x} \otimes 1^{\otimes k-i-1}+ & \\
\sum_{i_{1}+\cdots+i_{n}+1=k-l} 1^{\otimes i_{1}} \otimes x_{1} \otimes 1^{\otimes i_{2}} \otimes \cdots \otimes x_{l} \otimes 1^{\otimes i_{l+1}} & \text { if } k>l\end{cases}
\end{aligned}
$$

where $\underline{x}:=\mu_{l}\left(x_{1} \otimes \cdots \otimes x_{l}\right)$.

Remark 7.7. The compatibility relation (2) can be unified as follows: For any elements $x_{1}, \ldots, x_{m} \in \mathcal{H}$

$$
\Delta_{k} \circ \mu_{l}\left(x_{1} \otimes \cdots \otimes x_{l}\right)=\sum_{\mu_{k}\left(y_{1} \otimes \cdots \otimes y_{k}\right)=\mu_{l}\left(x_{1} \otimes \cdots \otimes x_{l}\right)} y_{1} \otimes \cdots \otimes y_{k} .
$$


Proposition 7.8. There exists a unique family of coproducts $\Delta_{m}, m \in \underline{n}$, such that

$$
\Delta_{m}(1)=1^{\otimes m}, \Delta_{m}(v)=\sum_{i=0}^{m-1} 1^{\otimes i} \otimes v \otimes 1^{\otimes m-i-1} \text { for all } v \in V
$$

on the free unital $\underline{n}$-magmatic algebra on $V$, which makes it into a connected unital $(\underline{n} ; \underline{n})$-magmatic bialgebra for which $V$ is primitive.

Proof. The existence is due to Example 7.5, where we identify the unit 1 with the empty tree $\emptyset$. The uniqueness is due to the compatibility relation. The proof is similar to the non-unital framework. Indeed, let us construct $\Delta_{m}: \operatorname{Mag}^{\underline{n}}(V) \rightarrow \operatorname{Mag}^{\underline{n}}(V)^{\otimes m}$, for all $m \in \underline{n}$ as a $\underline{n}$-magmatic coalgebra co-operation induced by:

$$
\begin{aligned}
& \Delta_{m}(1)=1^{\otimes m} \\
& \Delta_{m}(v)=\sum_{i=0}^{m-1} 1^{\otimes i} \otimes v \otimes 1^{\otimes m-i-1},
\end{aligned}
$$

and verifying the compatibility relation (2). It suffices to consider $V=\mathbb{K}$. As any tree $t \in \mathrm{PRT}^{\underline{n}} \backslash \mathrm{PRT}_{1}^{\underline{n}}$ can be uniquely viewed as the $k$-grafting of trees $t_{1}, \ldots, t_{k}$ with $t_{i} \neq \emptyset$ for all $i$ (for some $k>1$ ), the $m$-ary co-operation on $t$ evaluates to

$$
\Delta_{m}(t)=\Delta_{m} \circ \vee_{k}\left(t_{1} \otimes \cdots \otimes t_{k}\right) .
$$

Therefore the compatibility relation induces the value of $\Delta_{m}(t)$. This proves the uniqueness (there is no other choice to construct the $m$-ary co-operations). Note that the counit relation is verified. Indeed, let $t_{1}, \ldots, t_{k} \in \mathrm{PRT}^{n}$ non-empty, then:

$$
\begin{aligned}
\wedge_{m} \circ \vee_{k+1}(t_{1} \otimes \cdots \otimes \underbrace{\emptyset}_{(i)} \otimes \cdots \otimes t_{k}) & \sum_{\vee_{m}\left(s_{1} \otimes \cdots \otimes s_{m}\right)=\vee_{k+1}\left(t_{1} \otimes \cdots \otimes \emptyset \otimes \cdots \otimes t_{k}\right)} s_{1} \otimes \cdots \otimes s_{m} \\
= & \sum_{\vee_{m}\left(s_{1} \otimes \cdots \otimes s_{m}\right)=\vee_{k}\left(t_{1} \otimes \cdots \otimes t_{k}\right)} s_{1} \otimes \cdots \otimes s_{m} \\
= & \wedge_{m} \circ \vee_{k}\left(t_{1} \otimes \cdots \otimes t_{k}\right) .
\end{aligned}
$$

This proves that the counit relation is verified.

We get Prim $\operatorname{Mag}^{\underline{n}}(V)=V$ and the connectedness, with the following filtration:

$$
F_{r}(\operatorname{Mag} \underline{\underline{n}}(V))=\mathbb{K} 1 \oplus \bigoplus_{m=0}^{r} \mathbb{K}\left[\mathrm{PRT}_{m}^{\frac{n}{m}}\right] \otimes V^{\otimes m} .
$$

Lemma 7.9. Every $\underline{n}_{m}$-rooted magmatic algebra $\bar{A}$ admits a unit 1 , such that $A:=$ $\mathbb{K} 1 \oplus \bar{A}$ is equipped with $M a g_{\text {root } \backslash \underline{m}^{-o p e r a t i o n s . ~}}$

Proof. In the particular case where $(T ; S)=(\underline{m}, \underline{n})$, insertion of units into a $k$-ary operation from $M a g g_{\text {root } \backslash \underline{m}}^{n}$ yields $k^{\prime}$-ary operations from $M a g \frac{n}{r_{\text {root } \backslash \underline{m}}}$ with $k^{\prime}<k$. 
Proposition 7.10. There is a forgetful functor (in the unital case) given by

$$
\begin{gathered}
()_{M a g} \frac{n}{\text { root } \backslash \underline{m}}:\{\underline{n} \text {-magmatic algebra }\} \rightarrow\left\{\underline{n}_{\underline{m}} \text {-rooted magmatic algebra },\right\} \\
\left(A,\left\{\mu_{k}\right\}_{k \in\{2, \ldots, n\}}\right) \\
\left.\mapsto\left(A,\left\{\mu_{k} \circ \text { (operation from the } \underline{n} \text {-magmatic algebra }\right)\right\}_{k \in\{m+1, \ldots, n\}}\right) .
\end{gathered}
$$

Proof. The proof in the non-unital framework has to be completed by the verification of the unital relations. Indeed, the generating operations

$$
\left\{\mu_{k} \circ \text { (operation from the } \underline{n} \text {-magmatic algebra) }\right\}_{k \in\{m+1, \ldots, n\}}
$$

are again given by a right ideal of an $\underline{n}$-magmatic operad.

Next we focus on the construction of the universal enveloping functor in this context. Now, for $A$ a $\underline{n}_{\underline{m}}$-rooted magmatic algebra, we construct $\underline{n}$-magmatic algebra $U_{M a g_{\text {root } \backslash m}}(A)$ as the quotient of the free $\underline{n}$-magmatic algebra $\operatorname{Mag}^{\underline{n}}(A)$ by the relations which identify $\tilde{\mu}_{k} \circ(\ldots)$ with the corresponding operations in the free $\underline{n}$-magmatic algebra, and furthermore by the relations identifying the unitary relations $\widetilde{\mathcal{R}}$ verified in $A$ with the unitary relations $\mathcal{R}$ verified in $\operatorname{Mag}^{n}(A)$.

Proposition 7.11. The universal enveloping functor

$$
U_{M a g} \frac{n}{r o o t \backslash \underline{m}}:\left\{\underline{n}_{\underline{m}} \text {-rooted magmatic algebra }\right\} \rightarrow\{\underline{n} \text {-magmatic algebra }\}
$$

is left adjoint to the forgetful functor

$$
()_{M a g}{ }_{\text {root } \backslash \underline{m}}:\{\underline{n} \text {-magmatic algebra }\} \rightarrow\left\{\underline{n}_{\underline{m}} \text {-rooted magmatic algebra }\right\} .
$$

Proof. The proof is similar to the proof of Proposition 5.6 except that we must verify that the morphism $f: B \rightarrow(A)_{M a g \frac{n}{r o o t \backslash m}}$ passes through the quotient. Indeed passing it to the quotient gives the $\underline{n}$-magmatic algebra morphism $U_{M a g} \frac{\underline{n}}{\text { root } \backslash \underline{m}}(B) \rightarrow A$ as the image $\mathcal{R}\left(x_{1}, \ldots, x_{n}\right)$ and $\widetilde{\mathcal{R}}\left(x_{1}, \ldots, x_{n}\right)$ are the same, namely $\mathcal{R}\left(f\left(x_{1}\right), \ldots, f\left(x_{n}\right)\right)$ and $\widetilde{\mathcal{R}}\left(f\left(x_{1}\right), \ldots, f\left(x_{n}\right)\right)$.

Corollary 7.12. The universal enveloping functor of the free unital $\underline{n}_{\underline{m}}$-rooted magmatic algebra is isomorphic to the free unital $\underline{n}$-magmatic algebra:

$$
U_{M a g_{\text {root } \backslash \underline{m}}^{n}}\left(\operatorname{Mag}_{\text {root } \backslash m}^{n}(V)\right) \cong \operatorname{Mag}^{n}(V) \text {. }
$$

Theorem 7.13. Let $m, n \in \mathbb{N}_{\geqslant 2}$ with $m \leqslant n$. If $\mathcal{H}$ is a unital $(\underline{m} ; \underline{n})$-magmatic bialgebra over a field $\mathbb{K}$ of characteristic zero, then the following are equivalent:

(i) $\mathcal{H}$ is a connected unital $(\underline{m} ; \underline{n})$-magmatic bialgebra.

(ii) $\mathcal{H}$ is isomorphic to $U_{\text {Mag } g_{\text {root } \backslash \underline{m}}}(\operatorname{Prim} \mathcal{H})$ as a unital $(\underline{m} ; \underline{n})$-magmatic bialgebra.

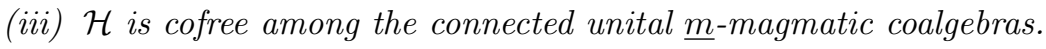

The proof is similar to the non-unital case treated above. 
Remark 7.14. Unital $S$-magmatic algebra, $S$-magmatic coalgebra, $(T ; S)$-magmatic bialgebra would be better described by pseudo-operads with a zero-ary operation and without associativity of composition for the cases where this operation is involved. The exploration of this generalized operadic setting is still open; compare BorisovManin [1].

\section{Combinatorics of the operads $M a g_{\text {root } \backslash T}^{S}$}

Lemma 8.1. The generating series $f_{M a g_{\text {root } \backslash T}^{S}}(x)=\sum_{n \geqslant 1} \operatorname{dim} M a g_{\text {root } \backslash T}^{S} x^{n}$ of the non-symmetric operad $M a g_{\text {root } \backslash T}^{S}$ is given by

$$
x+\sum_{k \in S \backslash T}\left(f_{M^{\prime} g^{S}}(x)\right)^{k},
$$

where $f_{M a g^{S}}(x)$ is the generating series of the non-symmetric operad $\mathrm{Mag}^{S}$.

Proof. The dimension of the space $\left(\operatorname{Mag}_{\text {root } \backslash T}^{S}\right)_{n}$ of operations with $n$ arguments is given by the number of planar trees with $n$ leaves, which fulfill the property that the arity $k$ of the root is an element of $S \backslash T$ and the arity of each other internal vertex is an element of $S$. Equivalently, every tree representing an operation in $M_{a g} g_{\text {root } \backslash T}^{S}$ is either the tree which consists only of the root, or is given by the choice of $k \in S \backslash T$ and an ordered $k$-tuple of planar reduced trees that represent operations in $\mathrm{Mag}^{S}$. Thus we get the formula $f_{M a g_{\text {root } \backslash T}^{S}}(x)=x+\sum_{k \in S \backslash T}\left(f_{M a g^{S}}(x)\right)^{k}$.

Proposition 8.2. Let $T \subset S \subset \mathbb{N}_{\geqslant 2}$. Then

$$
x+\sum_{k \in S \backslash T}\left(f_{M a g^{S}}(x)\right)^{k}=f_{M a g_{\text {root } \backslash T}^{S}}(x)=\left(x-\sum_{i \in T} x^{i}\right) \circ\left(x-\sum_{i \in S} x^{i}\right)^{\circ(-1)} .
$$

Proof. The first equality holds by Lemma 8.1. For the second equality, we may use Corollary 6.10 together with Proposition 3.8:

It follows from Corollary 6.10 that

$$
\left(f_{\text {Mag }^{T}}(x)\right)^{\circ(-1)} \circ f_{M^{S} S}(x)=f_{M_{\text {root }}^{S} T}(x) .
$$

By Proposition 3.8, $\left(f_{\text {Mag }^{T}}(x)\right)^{\circ(-1)}$ is given by $\left(x-\sum_{i \in T} x^{i}\right)$, and also $f_{M a g}(x)$ is the composition inverse of $\left(x-\sum_{i \in S} x^{i}\right)$. Hence the second equation follows.

Example 8.3. The Super-Catalan numbers (cf. [16, A001003]) are the coefficients of the generating series of $M a g^{\mathbb{N}} \geqslant 2$ :

$$
f^{M a g^{\mathrm{N}} \geqslant 2}(x)=\sum_{n \geqslant 1} C_{n} x^{n}=\frac{1}{4}\left(1+x-\sqrt{1-6 x+x^{2}}\right) .
$$

Let $S=\mathbb{N}_{\geqslant 2}$ and $T=\mathbb{N}_{\geqslant 2}-\{2\}=\{3,4, \ldots\}$. Then we obtain

$$
f_{M a g_{\text {root } \backslash T}^{S}}(x)=x+\sum_{k \in\{2\}}\left(f_{M a g^{\mathbb{N}} \geqslant 2}(x)\right)^{k}=x+\frac{(x-1)^{2}-(x+1) \sqrt{1-6 x+x^{2}}}{8} .
$$


In low degrees, this series is equal to

$$
x+x^{2}+2 x^{3}+7 x^{4}+28 x^{5}+121 x^{6}+550 x^{7}+\ldots .
$$

To verify the second equation in this case, we note that

$$
\begin{aligned}
\left(x-\sum_{i \in \mathbb{N} \geqslant 2} x^{i}\right)^{\circ(-1)} & =\left(\frac{x(1-2 x)}{1-x}\right)^{\circ(-1)} \\
& =\frac{1}{4}\left(1+x-\sqrt{1-6 x+x^{2}}\right) \\
& =f_{\text {Mag }^{\mathbb{N}} \geqslant 2}(x) .
\end{aligned}
$$

Since $\left(x-\sum_{i \geqslant 3} x^{i}\right) \circ f_{M a g^{\mathbb{N}} \geqslant 2}(x)=f_{M a g^{\mathbb{N}} \geqslant 2}(x)-\sum_{i \geqslant 3}\left(f_{M a g^{\mathbb{N}} \geqslant 2}(x)\right)^{i}$, we get the equation

$$
f_{M a g^{\mathbb{N}} \geqslant 2}(x)=f_{M a g_{\text {root } \backslash T}^{S}}(x)+\sum_{i \geqslant 3}\left(f_{M a g^{\mathbb{N}} \geqslant 2}(x)\right)^{i}=x+\sum_{k \geqslant 2}\left(f_{M a g^{\mathbb{N}} \geqslant 2}(x)\right)^{k}
$$

in this case.

Example 8.4. Let $S=\mathbb{N}_{\geqslant 2}$ and $T=\{2 k+1: k \geqslant 1\}$. Then we obtain by analogous computations that

$$
f_{M a g_{\text {root } \backslash T}^{S}}(x)=x-\frac{1+x^{2}-2 x-(x+1) \sqrt{1-6 x+x^{2}}}{(-7)+x^{2}-2 x-(x+1) \sqrt{1-6 x+x^{2}}} .
$$

In low degrees, this series is equal to

$$
x+x^{2}+2 x^{3}+8 x^{4}+32 x^{5}+140 x^{6}+640 x^{7}+\ldots .
$$

\section{Acknowledgements}

R. Holtkamp wants to thank for the hospitality of the Institut de Mathématiques et de modélisation de Montpellier. Both authors thank the Max-Planck-Institute for Mathematics Bonn, where this work was begun, for support. The authors thank Jean-Louis Loday for helpful discussions and his remarks on a first version of this paper. Both authors would like to thank the referee for helpful comments.

\section{References}

[1] D. Borisov and Y. Manin, Generalized operads and their inner cohomomorphisms, preprint; math.CT/0609748.

[2] E. Burgunder, Bigèbre magmatique et bigèbre Associative-Zinbiel, Mémoire de Master, Strasbourg, 2005.

[3] E. Burgunder, Infinite magmatic bialgebras, Adv. Appl. Math. 40(3) (2008), 309-329.

[4] P. Cartier, Hyperalgèbres et groupes de Lie formels, Séminaire Sophus Lie, 2e année: 1955/56, Faculté des Sciences de Paris.

[5] L. Gerritzen, Planar rooted trees and non-associative exponential series, Adv. Appl. Math. 33(2) (2004), 342-365. 
[6] V. Ginzburg and M. Kapranov, Koszul duality for operads, Duke Math. J. 76(1) (1994), 203-272.

[7] R. Holtkamp, On Hopf algebra structures over free operads, Adv. Math. 207(2) (2006), 544-565.

[8] R. Holtkamp, J.-L. Loday, and M. Ronco, Coassociative magmatic bialgebras and the Fine numbers, J. Algebraic Combin. 28(1) (2008), 97-114.

[9] J.-L. Loday, Generalized bialgebras and triples of operads, preprint, math. QA/0611885.

[10] J.-L. Loday and M. Ronco, On the structure of cofree Hopf algebras, J. Reine Angew. Math. 592 (2006), 123-155.

[11] J.-L. Loday and M. Ronco, Algèbres de Hopf colibres, C. R. Acad. Sci. Paris 337(3) (2003), 153-158.

[12] J. Milnor and J.C. Moore, On the structure of Hopf algebras, Ann. of Math. (2) 81(2) (1965), 211-264.

[13] D. Quillen, Rational homotopy theory, Ann. of Math. (2) 90(2) (1969), 205-295.

[14] M. Ronco, Eulerian idempotents and Milnor-Moore theorem for certain noncommutative Hopf algebras, J. Algebra 254(1) (2002), 152-172.

[15] M. Ronco, Primitive elements in a free dendriform algebra, New trends in Hopf algebra theory (La Falda, 1999), 245-263, Contemp. Math. 267, Amer. Math. Soc., Providence, RI, 2000.

[16] N. Sloane (Editor), The On-Line Encyclopedia of Integer Sequences, 2007, http://www.research.att.com/ njas/sequences/index.html.

[17] B. Vallette, A Koszul duality for props, Trans. Amer. Math. Soc. 359 (2007), 4865-4943.

Emily Burgunder burgunder@math.univ-montp2.fr

I3M, Université Montpellier II, 34095 Montpellier, France

Ralf Holtkamp ralf.holtkamp@ruhr-uni-bochum.de

Fakultät für Mathematik, Ruhr-Universität, 44780 Bochum, Germany 